and Feedback (A\&F) intervention to improve the quality and equity of care for ovarian cancer patients residing in Piedmont. This A\&F is part of the activities of the EASY-NET network program (https://easy-net.info/).

Methodology All consecutive patients treated for newly diagnosed EOC were included by 34 centres from May 2016 to September 2020. Clinical data were entered in a dedicated web database and data quality was centrally monitored. During the audit, 14 feedback meetings were organized with the participating centres to discuss data quality and preliminary results. The treating hospitals were classified according to the mean yearly volume of surgical activity $(\geq 35 ; 34-18 ;<18$ patients). Adherence to previously selected structure, process and outcome indicators were analysed by volume of activity of the centre and semester of enrolment. Adherence was classified as: high (>75\%), medium (75-60\%) and low $(<60 \%)$. Overall survival (OS) was analysed with a multivariable Cox model including prognostic factors, hospital volume of activity and level of adherence to process indicators.

Result(s)* The present analysis includes 905 patients with EOC diagnosed until December 2019 (23.4\% early stages, $76.6 \%$ advanced). Out of 12 analysed indicators, 4 showed a high level of adherence (e.g., Completeness of diagnosis and staging: 83\%), 3 a medium level (e.g., Adherence to surgical guidelines: $65.5 \%$ ) and 5 a low level (e.g., Timing and number of cycles for NACT: 57.1\%). In general, there was a lower adherence to guidelines by centres with a low volume of activity. For most of the indicators there was an improvement over time. Adherence to guidelines was associated to better OS after adjustment for prognostic factors.

Conclusion* The A\&F intervention was useful to support the identification of reference centres, to promote centralization, to reduce variability among regional hospitals and to increase the appropriateness of treatment. Adherence to guideline recommendations was associated to a better outcome.

The EASY-NET project was funded by Ministry of Health and participating Regions (NET-2016-02364191).

\section{HOW TO BREAKING BAD NEWS: AN INTERNATIONAL SURVEY AMONG PHYSICIANS AND MEDICAL STUDENTS IN 1146 PARTICIPANTS}

${ }^{1,2} \mathrm{~J} \mathrm{Sehouli}^{*},{ }^{3} \mathrm{~A}$ Pirmorady, ${ }^{2} \mathrm{~S}$ Boz, ${ }^{4,5} \mathrm{~K}$ Hasan, ${ }^{1,2} \mathrm{~K}$ Pietzner, ${ }^{6} \mathrm{E}$ Petru, ${ }^{7} \mathrm{~V}$ HeinzelmannSchwarz, ${ }^{1} \mathrm{E}$ Roser, ${ }^{1} \mathrm{D}$ Dimitrova, ${ }^{1} \mathrm{E}$ Herzog. ${ }^{1}$ Charité Universitätsmedizin Berlin, Klinik für Gynäkologie, Campus Virchow Klinikum, Berlin, Germany; ${ }^{2}$ Noggo e.V., Nord-Ostdeutsche Gesellschaft für Gynäkologische Onkologie, Berlin, Germany; ${ }^{3}$ Charité Universitätsmedizin Berlin, Medizinische Klinik mit Schwerpunkt Psychosomatik, Berlin, Germany; ${ }^{4}$ MVZ Nazarethkirchstraße, Berlin, Germany; ${ }^{5}$ AMBOSS GmbH, Berlin, Germany; ${ }^{6}$ Univ. Klinik für Frauenheilkunde und Geburtshilfe, Medizinische Universität Graz, Klinische Abteilung für Gynäkologie, Graz, Austria; 'Universitätsspital Basel, Gynäkologie/Gynäkologische Onkologie Frauenklinik, Basel, Switzerland

\subsection{6/ijgc-2021-ESG0.313}

Introduction/Background* Delivering bad news to patients is one of the most challenging everyday tasks in medical practice. Despite its high relevance for patients, relatives, and medical staff, there is lacking data about training, experience, expectations and preferences of physicians and medical students on breaking bad news.

Methodology We therefore conducted an international survey in Germany, Switzerland and Austria using an online questionnaire among physicians and medical students. Recorded data includes demographics, physician and medical student's clinical experience and their training and educational preferences.

Result(s)* A total of 831 physicians, including 25\% gynecologists, 23.1\% internal specialists and 315 medical students have participated in the survey. Physicians stated that $45,2 \%$ deliver bad news several times a week and $32.6 \%$ several times a month. Difficulties controlling their emotions was declared by $37 \%$ of the participants. The median score concerning fear was 3.5 on a scale of 0 to 10 ; for medical students the median score was 5.2. When delivering bad news, $70.4 \%$ of the physicians are generally alone with the patient; only $57.4 \%$ encouraging patients to be accompanied by their friends or relatives. Among the physicians, only 31.2\% mentioned having learned adequate communication skills. Almost all physicians stated that communication with patients has a significant impact on their employee satisfaction. Therefore, a need for systematic training and education in breaking bad news exists. The most preferred educational tools were seminars with simulation patients $(53.3 \% / 79.3 \%)$, learning from supervisors (59.7\%/64.1\%) and systematic supervision (48.9\%/ $40.4 \%)$ for physicians and medical students respectively. Digital tools may help in preparing individual sessions $(54.6 \%)$ and for debriefing $(38.8 \%)$ in the clinical day practice. Missing awareness (52.5\%), costs (35.1\%) and limited time $(10.3 \%)$, were the largest barriers for communication education.

Conclusion* We could demonstrate the high need for more education and training in breaking bad news communication skills among physicians and medical students. Hospitals, authorities, medical schools and post-graduate training programs are strongly encouraged to fill this gap.

\section{IMPACT OF COVID PANDEMIC IN THE DIAGNOSIS OF ENDOMETRIAL AND OVARIAN CANCERS}

JM Sole-Sedeno*, E Miralpeix Rovira, S Espuelas, J Castella, B Fabrego, A Salvado, G Mancebo. Hospital del Mar, Gynecology and Obstetrics, Barcelona, Spain

\subsection{6/ijgc-2021-ESG0.314}

Introduction/Background* The COVID pandemic has had the collateral effect of an alteration in the diagnosis and care of other diseases. In our center, a 30\% decrease in the number of cancer diagnoses has been estimated. The objective of this study was to account for this variation in the area of gynecological oncology.

Methodology The diagnoses of endometrial cancer and ovarian cancer, of any histology, diagnosed in our center between April 1, 2019 and March 31, 2021 were reviewed. Data were compared between the pre-COVID period (1/04/19-31/03/20) and COVID (01/04/20-31/03/21).

Result(s)* The number of endometrial cancer diagnoses decreased from 33 to 25 cases (25\% decrease). Grouped by stages, initial diagnoses (FIGO I/II) went from $72 \%$ to $64 \%$, with an increase in advanced stages (FIGO III/IV) $(27 \%$ to $36 \%)$.

Regarding ovarian cancer, the number of diagnoses was similar (24 pre-COVID vs 26 COVID), although with a slight increase in advanced stages (58\% pre-COVID vs 65\%). Within the advanced ones, we observed a significant increase in their severity, being in COVID time all stages III (14 cases), while in COVID time $11.2 \%$ were IVA stages and $41.2 \%$ IVB. 
Conclusion* In neoplasms where the initial symptoms are mild, such as endometrial cancer, the number of cases has been reduced, which may be due to the lack of consultation by patients. Regarding ovarian cancer, we have not seen a decrease in cases, although we have seen an increase in the stage. It is expected that the consequences of the COVID pandemic will continue to be felt in the future with an increase in the advanced stages of these neoplasms.

\section{ACS NSQIP - PERSONALISED RISK PREDICTION TOOL FOR POSTOPERATIVE COMPLICATIONS IN GYNAEONCOLOGY SURGERY?}

${ }^{1} \mathrm{~L}$ Sevinyan*, ${ }^{2} \mathrm{~S}$ Jones, ${ }^{3} \mathrm{~J}$ Horne, ${ }^{3} \mathrm{R}$ Bharathan, ${ }^{1} \mathrm{~A}$ Tailor, ${ }^{1} \mathrm{~S}$ Butler-Manuel, ${ }^{4} \mathrm{P}$ Williams, ${ }^{1,5}$ T Madhuri. ${ }^{1}$ Royal Surrey County Hospital, Gynaeoncology, Guildford, UK; ${ }^{2}$ Cardiff University, UK; ${ }^{3}$ University Hospitals of Leicester, UK; ${ }^{4}$ University Of Surrey, UK; ${ }^{5}$ University Of Brighton, UK

\subsection{6/ijgc-2021-ESG0.315}

Introduction/Background* Despite the informed consent process, patients' understanding of potential post-operative complications is often limited, making it difficult to call the decision an informed one, so estimating the risk of postoperative complications is important for shared decision making and to help multidisciplinary teams plan postoperative care. Increased incidence of gynaecological cancers and operations, especially technically challenging minimally invasive surgery (MIS) in older, obese and patients with multiple comorbidities, requires accurate prediction of the likelihood of mortality and morbidity and patient involvement in joint decision making about the management. ACS-NSQIP (American College of Surgeons National Surgical Quality Improvement Program) risk calculator is a validated web-based tool based on 21 preoperative risk factors to predict 8 post-operative outcomes. The objective of our study was to explore the validity of ACS NSQIP in gynaeoncology for perioperative prediction of postoperative complications.

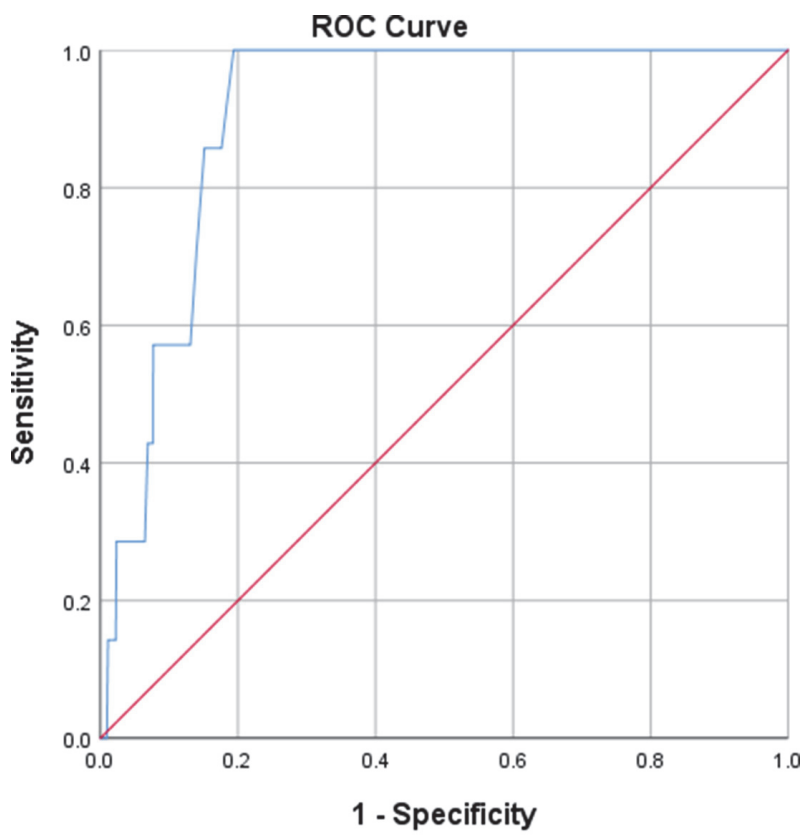

Diagonal segments are produced by ties
Methodology A retrospective multicentre cohort study evaluated 1552 patients who underwent surgery at a tertiary oncology centre. Data collection undertaken through dedicated database. Data collated on 764 patients undergoing robotic, 248 laparoscopic and 540 open surgery for suspected or confirmed gynaecological malignancy. All missing data collated from patient notes. Following data lock with the actual postop event/complication that occurred in this retrospective cohort, ACS NSQIP used to count predictive scores for each patient. Data analysis evaluating ACS-NSQIP validity and relevance in gynaecological oncology patients and its ability to predict postoperative complications performed.

Result(s)* ACS-NSQIP was found to best predict mortality (AUC - 0.908), it was most accurate for prediction of complications as follows: discharge to rehabilitation (AUC-0.875), cardiac complications (AUC-0.854), sepsis (AUC-0.795), pneumonia (AUC-0.764), return to theatre (AUC-0.696), surgical site infection (AUC-0.686), VTE (AUC-0.676), readmission (AUC-0.669), renal failure (AUC-0.601). Poor result in the prediction of UTI (AUC-0.543) was noted.

Conclusion* ACS-NSQIP risk calculator appears to predict major complications and post-operative mortality making it useful as an informed consent tool. Preliminary data suggests that further validation is required to fully evaluate if the risk scores may be used to inform patients pre-operatively of their risk of complications and is currently being undertaken.

\section{APPLICABILITY OF PRE-OPERATIVE PATIENT REPORTED DUKE ACTIVITY SCALE INDEX IN PREDICTION OF POSTOPERATIVE COMPLICATIONS IN GYNAECOLOGICAL ONCOLOGY}

${ }^{1}$ L Sevinyan*, 'A Tailor, ${ }^{2} \mathrm{P}$ Prabhu, ${ }^{3} \mathrm{P}$ Williams, ${ }^{1,4} \mathrm{~T}$ Madhuri. ${ }^{1}$ Royal Surrey County Hospital, Gynaeoncology, UK; ${ }^{2}$ Royal Surrey County Hospital, Anaesthetics, UK; ${ }^{3}$ University Of Surrey, UK; ${ }^{4}$ University Of Brighton, UK

\subsection{6/ijgc-2021-ESGO.316}

Introduction/Background* Increase in the incidence of gynaecological cancers has resulted in increased operative procedures, specifically in patients with multiple comorbidities including obesity and frailty. This is often associated with prolonged admission and higher rates of postoperative mortality and morbidity and presents a challenge with an unmet need for an accurate, personalised risk prediction. Duke Activity Scale Index (DASI) is a 12 item scale in the form of selfreported questionnaire based around commonly performed activities of daily living. Currently, DASI is used to evaluate patients with cardiovascular diseases, however there is growing interest in utilising it in preoperative setting in different specialities. This study investigates the accuracy of DASI in preoperative prediction of postoperative outcomes in gynaeoncology.

Methodology A retrospective cohort study of 486 patients who had undergone an operative treatment at a tertiary oncology centre. Data collection undertaken through dedicated gynaeoncology database and missing data collected through patients' records. All patients had completed the DASI questionnaire prior to their consultation. Actual postoperative 30 day complications and the length of stay also recorded. DASI was then compared with the occurrence of postoperative complications.

Result(s)* 242 patients had a Da Vinci robot-assisted procedure, 44 - laparoscopic and 200 - open surgery. 133/486 were 